\title{
A Review on Self Micro Emulsifying Drug Delivery System: An Approach to Enhance the Oral Bioavailability of Poorly Water Soluble Drug
}

\author{
Mamta Patil *1, Shobha Palde ${ }^{2}$, Amol Deshmukh ${ }^{3}$ \\ SMBT College of Pharmacy, Nandi-Hills, Dhamangaon, Nashik, Maharashtra, India.
}

\begin{abstract}
A B S T R A C T
Self-micro emulsifying drug delivery framework is the one of the methodology for improving the solvency of the hydrophobic medication. The medications which are insoluble in water can be formulated in this technique by solubilizing it in the lipid vehicle to absorb through the membrane. The lipid and surfactants are utilized to build the solvency of the drug and improve absorption. This improves the dissolution rate of the drug by expanding its solubility. A large number of the obstacles and arrangements are depicted in this article. This system picked up consideration as it likewise improves the bioavailability of the drug. This article has total survey about SMEDDS for present work and for future viewpoint too.
\end{abstract}

Keywords: Self-microemulsifying, surfactant, bioavailability, absorption, ternary phase diagram.

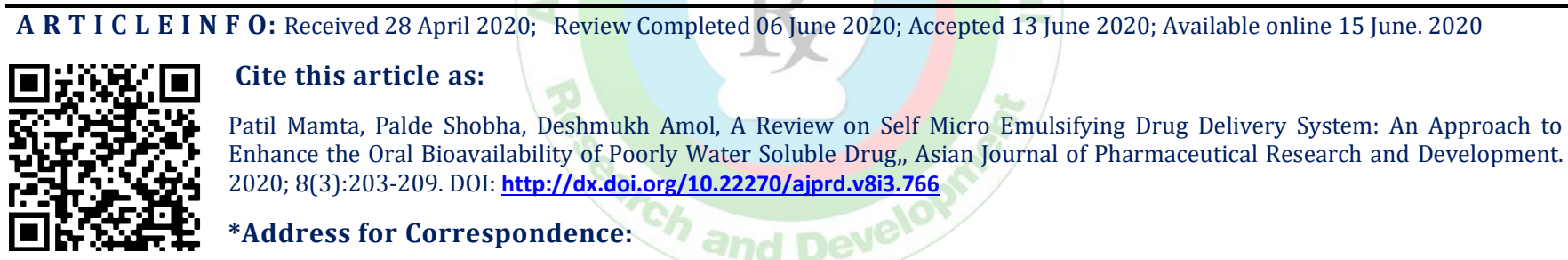

Patil M, SMBT College of pharmacy, Nandi hills, Dhamangaon, Nashik, Maharashtra, India.

\section{INTRODUCTION:}

$\mathrm{T}$ Today, one of the important problems to drug method development is poor water solubility of latest drug. More than forty\% of all new capsules are poorly water soluble ${ }^{1}$. Poor solubility and ultimately low dissolution charge of these tablets within the gastrointestinal fluids cause terrible bioavailability ${ }^{14-23}$. There are several strategies for development in bioavailability which include use of crystal polymorphism, surfactants, salt formation, pulverization, length reduction of debris, strong dispersion, microemulsion, liposomes, complicated formation, Nano-particles, Nano and micro-spheres, use of pro-drugs and use of permeation enhancer. Recently, guidance of formulations with lipid base to upsurge the oral bioavailability of medication with terrible aqueous solubility is in fashion. Self-dispersing lipid formulations are labeled into two classes:

- Self-emulsifying drug shipping system (SEDDS) and
- Self-microemulsifying drug shipping machine

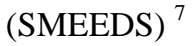

SMEDDS 6Y(Type III B structures) are defined as isotropic combos of natural or synthetic oils, stable or liquid surfactants, or one or more hydrophilic solvents and co-solvents/surfactants that have ability to shape satisfactory oil -in-water $(\mathrm{o} / \mathrm{w})$ microemulsions upon slight agitation followed by means of dilution in aqueous media, which include GI fluids ${ }^{14}$. These systems are developed through the use of a lipid provider which improves the gastro intestinal absorption of poorly water soluble capsules, permits the drug to stay in dissolved kingdom by shielding the drug from enzymatic response, thermodynamically stable, without difficulty manufactured and appropriate for oral drug transport ${ }^{18}$. The feature of oil on this system is to solubilize the lipophilic drug to be able to improve the drug loading and bioavailability. Medium chain triglycerides are maximum normally used as they're proof against precipitation. Hydrophobic drugs are easily CODEN (USA): AJPRHS 
solubilized in oil. As solubility is confined in oils, micro emulsification of oil and surfactant is hired which enhances the drug solubility in oils. Enhancement of drug solubility primarily depends on elements including efficiency and rapidity to micro emulsifies the chosen oil, solubility of drug in surfactant. Nonionic surfactants are normally desired in components as they have less ${ }^{15-21}$ CMC cost, they're much less poisonous, offers a greater emulsion stability over a wide variety of $\mathrm{pH}$ and ionic strength. Concentration of co-surfactant plays a chief position in lipid based totally formulation. Selection of surfactant and co-surfactant is necessary for the solubilization of drug. Organic solvents such as ethanol, propylene glycol, polyethylene glycol are appropriate for oral drug transport 18.

As oral direction has continually been favored and has ruled over different routes of administration due to its comfort, non-invasiveness, and cost effectiveness thus it turn out to be essential that drug must have some aqueous in addition to a few lipid solubility for higher absorption thru this path. The oral course isn't always suitable for those chemical entities which showcase terrible aqueous solubility ${ }^{21}$. Oral delivery of bad water soluble drugs the usage of lipid as automobile is a brand new and recent approach to conquer the aforesaid issues ${ }^{1}$.

There are number of formulation strategies that could be used to improve bioavailability of class IIdrugs, either by increasing the dissolution rate/ by presenting the drug in solution and maintaining the drug in solution in the intestinal lumen. As shown below in figure 1 . Bioavailability of class IV drugs can be improved by attention to the formulation. Formulation may improve bioavailability of class IV drugs but they are likely to be compromised by their poor membrane permeability. If a class II drug can be maintained in a solubilize state in the lumen of the gut one can achieve an absorption profile more like that of a class I drug. Formulation strategies can do little to improve the absorption of class IV and III drugs which are limited by poor membrane Permeability ${ }^{15}$

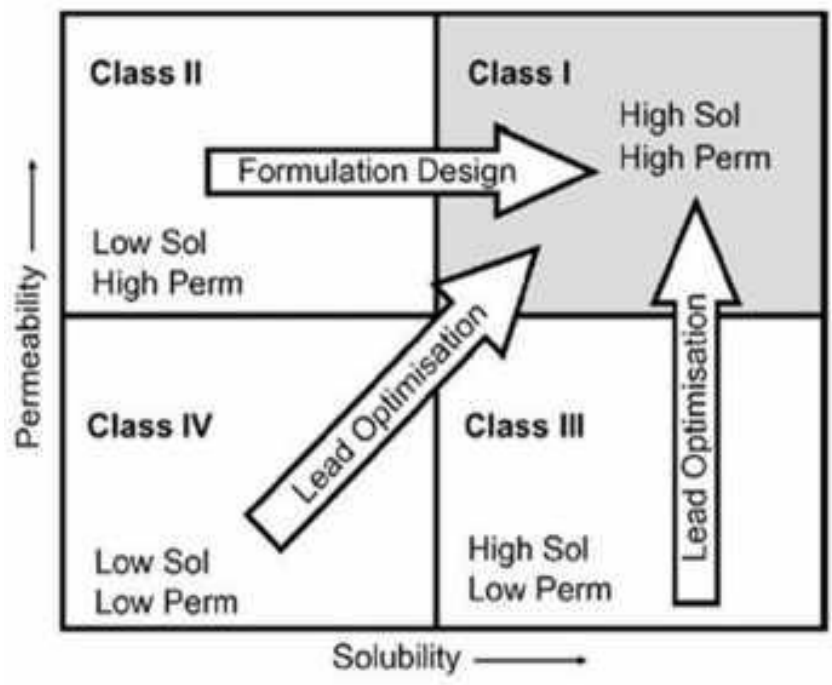

Figure 1: A Typical Representation of Biopharmaceutical Classification System ${ }^{15}$
Table 1: Summarizes Examples of Drugs Related to II, III and IV Classes

\begin{tabular}{|l|l|l|l|}
\hline Class I & Class II & Class III & Class IV \\
\hline Amiloride & Artemether & Abacavir & Albendazole \\
Abacavir & Dapsone & Allopurinol & Indinavir \\
Captopril & Carbamazepine & Ethambutol & Acetazolamide \\
Diazepam & Folic acid & Biperiden & Furosemide \\
Diltiazem & Greciofulvin & Captopril & Mesylate \\
Ethambutol & Ibuprofen & Metformin & Nelfinavir \\
Ketorolac & Itraconazole & Hydrochloride & \\
Levodopa & Febuxostat & Cementidine & \\
& & Atropine & \\
& & Sulphate & \\
\hline
\end{tabular}

\section{MECHANISM}

The unfastened energy of traditional emulsion may be very high as high strength is needed to form new surface among immiscible stages like oil andwater.Due to excessive free power,the emulsion might not be strong and the two levels tendto separate with time to reduce the interfacial vicinity and, in the end, the emulsion is stabilized via emulsifying retailers, which shape a monolayer of emulsion droplets, and subsequently reduces the interfacial electricity, as well as imparting a barrier to prevent coalescence ${ }^{1,13,21}$. Free electricity of formation could be very low and advantageous or even terrible which results in spontaneous emulsification in case of SMEDDS ${ }^{2,7,14,21}$.

Self-emulsification happens when the entropy trade that favors dispersion is greater than the power required to growth the floor place of the dispersion $1,3,7,14,15,21$. Hence for emulsification take region, it's far vital for the interfacial shape to no resistance in opposition to surface shearing ${ }^{21}$.The interface between the oil and aqueous non-stop phases is fashioned upon addition of a binary aggregate (oil/non-ionic surfactant) to water. This is followed by solubilization in the oil section, as a result of aqueous penetration through the interface. This occurs upto the solubilization limit attained near the interphase. Aqueous penetration will cause the formation of the dispersed liquid crystal (LC) section ${ }^{1,7,14}$. Lastly, the whole thing that is close to with the interface will be liquid crystal, the actual quantity of which relies upon upon the emulsifier awareness within the binary mixture. Hence, following mild agitation of the self-emulsifying gadget, Water swiftly penetrates into the aqueous cores main to interface disruption and droplet formation. This LC section is taken into consideration to be responsible for the excessive stability of the ensuing microemulsion in opposition to coalescence $^{14,21}$.

The unfastened strength of the traditional emulsion is an immediate feature of the power required to create a new surface between the water and oil stages and may be described with the aid of the equation: ${ }^{10}$

$\Delta \mathrm{G}=\Sigma \mathrm{Ni} \pi \mathrm{ri} 2 \mathrm{~S}$

Where, 
$\Delta \mathrm{G}$ - loose energy accompanying the system (aside from the loose energy of blending),

$\mathrm{N}$ - Total number of droplets,

$\mathrm{r}$ - Radius of the droplets,

$\mathrm{S}$ - Energy on the interface ${ }^{17,13,15,21}$

From this equation, it's far evident that the decreases the interfacial power the lower the loose power.

\section{EXCIPIENTS USED IN SMEDDS:}

Pharmaceutical worthiness of excipients and the toxicity issues of the segments utilized make the choice of excipients extremely basic. There is an incredible limitation as which excipients to be utilized. Early investigations uncovered that the self-micro emulsification process is explicit to the idea of the oil/surfactant pair, the surfactant concentration and oil/surfactant proportion, the fixation and nature of co-surfactant and surfactant/co-surfactant proportion and the temperature at which self-micro emulsification happens. These significant disclosures were additionally bolstered by the way that truth be told, quite certain blends of pharmaceutical excipients prompted productive self-microemulsifying frameworks ${ }^{17}$

\section{Oil/Lipid stage:}

The capacity of oil stage in self-micro emulsifying framework is to solubilize the hydrophobic/lipophilic dynamic moiety so as to improve both medication stacking and bioavailability of the hydrophobic dynamic moiety ${ }^{13}$. Oil solubilizes the hydrophobic medication and helps in self-emulsification. Lipid tends to build the division of medication moved by means of intestinal lymphatic framework and accordingly expanding lipophilic medication ingestion from the GI tract ${ }^{14}$. The principle standard for choosing the oil is that the medication ought to have high solvency in it so this will limit the volume of the plan for the conveyance of successful portion. Lipid part of SMEDDS definition shapes the center of emulsion molecule and is commonly made out of non-polar lipids. Long chain triglycerides (LCTs) and Medium chain triglyceride (MCTs) oils with various level of immersion have been utilized as oil stage in the definition of SMEDDS ${ }^{21}$. The grouping of oil present in SMEDDS is about the $40-80 \%$ the altered and hydrolyzed vegetable oils generally on the grounds that they show the greater solvency and great self-emulsifying property ${ }^{15}$. Edible oils are not every now and again chose because of their poor capacity to break down a lot of lipophilic medications. Altered or hydrolyzed vegetable oils have been generally utilized since these excipients for good emulsification frameworks with an enormous number of surfactants endorsed for oral organization and display better medication dissolvability properties ${ }^{12,19,24}$.

\section{Surfactant:}

Several mixes displaying surfactant properties might be utilized for the plan of self-emulsifying frameworks, yet the decision is restricted as not many surfactants are orally adequate. The most broadly suggested ones being the nonionic surfactants with a moderately high hydrophiliclipophilic equalization (HLB). Safety is a significant deciding component in picking a surfactant. These surfactants have a constrained self-emulsification limit. Non-ionic surfactants are less poisonous than ionic surfactants yet they may prompt reversible changes in the permeability of the intestinal lumen. Usually the surfactant concentration in the range of $30 \%$ and $60 \% \mathrm{w} / \mathrm{w}$ so as to shape stable SMEDDS. It is essential to decide the surfactant concentration appropriately as a lot of surfactants may cause GI irritate ${ }^{21,24}$.the four primary gatherings of surfactants are characterized as follows (Khoo, 1998) ${ }^{19}$.

- Anionic Surfactants: where the hydrophilic bunch conveys a negative charge, for example, carboxyl (RCOO-), sulphonate (RSO3-) or sulfate (RO-SO3-). Models: Potassium laurate, sodium lauryl sulfate.

- Cationic surfactants: where the hydrophilic gathering conveys a positive charge. Model: quaternary ammonium halide.

- Ampholytic surfactants: (additionally called zwitterionic surfactants) contain both a negative and positive charge. Model: sulfobetaines.

- Non-ionic surfactants:Where the hydrophilic gathering conveys no charge however infers its water solvency from exceptionally polar gatherings, for example, hydroxyl or polyoxyethylene (OCH2CH2O). Models: Sorbitan Ester (Spans), Polysorbates (Tweens) $)^{3,11,15,16,19}$

\section{Co-surfactants:}

The creation of an ideal SMEDDS requires moderately high fixations (by and large over 30\% w/w) of surfactants yet it causes GI aggravation. So co surfactant is utilized to diminish convergence of surfactant. Job of the co-surfactant together with the surfactant is to bring down the interfacial strain to a little even transient negative worth. At this worth the interface would extend to shape fine scattered beads, and hence adsorb more surfactant and surfactant/cosurfactant until their mass condition is sufficiently drained to make interfacial strain positive once more.

This procedure known as 'unconstrained emulsification' frames the micro emulsions. Natural solvents, appropriate for oral organization \{ethanol, propylene glycol (PG), polyethylene glycol (PEG), etc may assist with dissolving a lot of either the hydrophilic surfactant or the medication in the lipid base and cangoabout as co-surfactant in oneself emulsifying drug conveyance systems ${ }^{12,19,20}$.

\section{Co-solvents:}

Organic solvents, reasonable for oral organization (ethanol, propylene glycol (PG), polyethylene glycol (PEG), and so forth.) may assist with dissolving a lot of either the hydrophilic surfactant or the medication in the lipid base and can go about as co-surfactant in oneself emulsifying drug conveyance frameworks, in spite of the fact that alcohol free self-emulsifying microemulsions have additionally been depicted in the writing. Without a doubt, such frameworks may display a few focal points over the past definitions when consolidated in case dose structures, since alcohol and other unpredictable co-solvents in the ordinary self-emulsifying details are known to relocate into the shells of delicate gelatin or hard fixed gelatin containers bringing about the precipitation of the lipophilic medication. Then again, the lipophilic medication disintegration capacity of the alcohol free detailing might 
be constrained. Henceforth, appropriate decision must be made during choice of segment $2,3,11,22$.

\section{ADVANTAGES}

\section{Oral Bioavailability Improvement:}

Dissolution rate dependent ingestion is a central point that restrains the bioavailability of different ineffectively water dissolvable medications. The capacity of SMEDDS to introduce the medication to GIT in solubilized and micro emulsified structure (globule size between 1-100 nm) and resulting increment in explicit surface territory empower progressively productive medication transport through the intestinal watery limit layer and through the absorptive brush outskirt film prompting improved bioavailability. For example If there should be an occurrence of halofantrine around 6-8 crease increment in bioavailability of medication was accounted for in contrast with tablet detailing $^{3}$.

\section{Ease of assembling and scale-up:}

Ease of production and scale-up is one of the most significant favorable position that makes SMEDDS extraordinary when contrasted with other medication conveyance frameworks like strong scatterings, liposomes, nanoparticles, and so forth., managing improvement of bioaccessibility. SMEDDS require straightforward and affordable assembling offices like basic blender with fomenter and volumetric fluid filling hardware for large scale $^{3}$.

\section{Inter-subject and intra-subject inconstancy nourishment impacts are diminished:}

There are a few medications which show huge between subject and intra-subject variety in ingestion prompting diminished execution of medication and patient resistance. Food is a central point influencing the helpful execution of the medication in the body. SMEDDS are a valuable for such medications. A few investigations indicating that, the presentation of SMEDDS is free of nourishment and, SMEDDS offer reproducibility of plasma profile are accessible.

\section{Ability to convey peptides:}

One remarkable property that makes SMEDDS better as analyzed than the other medication conveyance frameworks is their capacity to convey macromolecules like peptides, hormones, chemical substrates and inhibitors as they offer insurance from enzymatic hydrolysis. The intestinal hydrolysis of prodrug by cholinesterase can be ensured if polysorbate 20 is emulsifier in micro emulsion definition. These frameworks are shaped suddenly without help of vitality or warming in this manner, appropriate for thermolabile medications, for example, peptides.

\section{Not affected by lipid absorption process:}

Unlike the other lipid-based medication conveyance frameworks, the exhibition of SMEDDS isn't impacted by the lipolysis, emulsification by the bile salts, activity of pancreatic lipases and fixed micelle arrangement. SMEDDS are not really processed before the medication is retained as they present the medication in micro emulsified structure which can undoubtedly enter the mucin and water unstirred layer.

\section{Increased Drug Loading Capacity:}

SMEDDS additionally give the upside of expanded medication stacking limit when contrasted and customary lipid arrangement as the dissolvability of ineffectively water dissolvable medications with moderate segment coefficient $(2<\log \mathrm{P}<4)$ are regularly low in characteristic lipids and a lot more prominent in amphiphilic surfactants, co surfactants and co-solvents ${ }^{5}$.

\section{DISADVANTAGES}

- Lack of good predicative in-vitro models for appraisal.

- High surfactant concentration (=30-60\%) irritates.

- Volatile co-solvents in the traditional definitions move into the shells of soft or hard gelatin capsule bringing about precipitation of lipophilic medications.

- Lack of good IVIVC correlation and appropriate animal model for in-vivo study ${ }^{15,16}$.

\section{DRUG PROPERTIES APPROPRIETES FOR SMEDDS}

- Portion dose not be so high

- Medication ought to be oil dissolvable

- High melting point medicate is inadequately fit to SMEDDS

- $\log$ P Value should not to be high ${ }^{22}$.

- Solvency: The medication ought to be insoluble in water. The $\log \mathrm{P}$ ought to be more noteworthy than 4 (lipophilic), high dissolvability in LCT for lymphatic assimilation $(>50 \mathrm{mg} / \mathrm{ml})$. BCS class II and IV ${ }^{19}$

- Low dose: To expand the bioavailability of the medication and to diminish molecule size, the portion ought to be low mainly<40mg.

- Poor BA: The bioavailability ought to be low as to improve the dissolvability of the medication

- Low melting point: The melting point ought to be low for the better absorbability of the medication.

- Ought to be chemically and physically stable in SMEDDS ${ }^{8}$.

\section{FORMULATTION OF SMEDDS:}

Various delivery systems for the lipophilic drugs are available such as, microemulsion, lipid solution, lipid emulsion, dry emulsion, whose formulation involve large number of possible combination of excipients, further to understand these lipid based formulation and to get a clear picture of all these different systems a particular classification system have been established called as 'lipid formulation classification system' have been introduced. The classification helps to better understand the fate of different lipid formulation in vivo. According to the composition and the effect of dilution and digestion on the ability to prevent precipitation of drug, lipid based formulations are classified into four groups: ${ }^{21}$. 


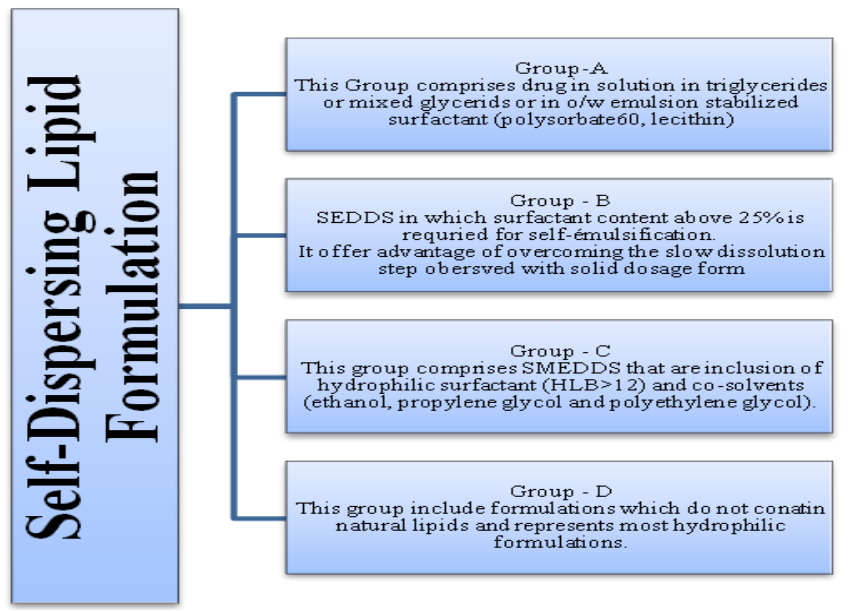

Figure 2: Flow chart for Self-Dispersing lipids Formulation .

The manufactured hydrophilic oils and surfactants give great dissolvability to hydrophobic medications than traditional vegetable oils. Ethanol, PG and PEG likewise contribute for the improvement of medication dissolvability in lipid vehicle ${ }^{16}$.

The accompanying focuses ought to be considered in the formulation of a SMEDDS -

- Find solvency of the medication in various oil, surfactants and surfactant.

- Determine dissolvability by including overabundance measure of medication in little vials containing $2 \mathrm{ml}$ of chose oil, surfactant and surfactant independently. The medication was blended in with glass rod for $30 \mathrm{~min}$, and afterward the vials saved for sonication around 2 hours. The vials are firmly plug and consistently mixed for 72 hours in orbital shaking incubator at $25^{\circ} \mathrm{C}$. Then centrifuged at $3500 \mathrm{rpm}$ for $20 \mathrm{~min}$. The $1 \mathrm{ml}$ supernatants are isolated and break down in methanol or alcohol and dissolvability is evaluated by UV-spectrophotometer at explicit frequency after proper dilution with methanol or alcohol. In any case, after dilution the arrangements are not satisfactory. So oils ought to be diluted with $66 \% \mathrm{v} / \mathrm{v}$ chloroform in methanol and surfactant ought to be diluted with $7 \%$ $\mathrm{v} / \mathrm{v}$ chloroform in methanol ${ }^{4}$.

- Select oil, surfactant and co surfactant dependent on the dissolvability of the drug.

- $\quad$ Select proportion of surfactant to co surfactant: The emulsifying impact is acceptable if the proportion of the surfactant to the co-surfactant is higher than 1:2.5 however stability properties are second rate at this proportion. Fixing the surfactant/co-surfactant proportion at $1: 1$ is a superior decision for the stability of SMEDDS ${ }^{4,14}$.

\section{- Preparation of phase diagram:}

- Phase conduct of each SMEDDS is concentrated cautiously by utilizing the phase graph. It is one of the significant attributes of SMEDDS to show the progressions when the framework is diluted, which may cause medicate precipitation. Thusly, phase behavior of each SMEEDS ought to be painstakingly examined. In light of dissolvability appeared by tranquilize in changed proportions of surfactants the ternary charts were created ${ }^{4,18,23,25}$.

- STEP A: -Solubilizing an inadequately water dissolvable medication and additionally pharmaceutical ingredient, in a blend of surfactant, co surfactant solvent. Presently blend the oil stage appropriately arranged, if necessary, by warming or other preliminary methods, to the solubilized drug formulation and altogether blended ${ }^{6}$.

- STEP B: - The emulsion would then be able to be added to a reasonable dosage form, for example, soft or hard-filled gelatin capsule and permitted to cool ${ }^{9}$.

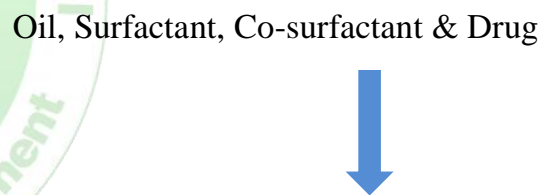

Drug is dissolved completely at ambient temperature with constant agitation

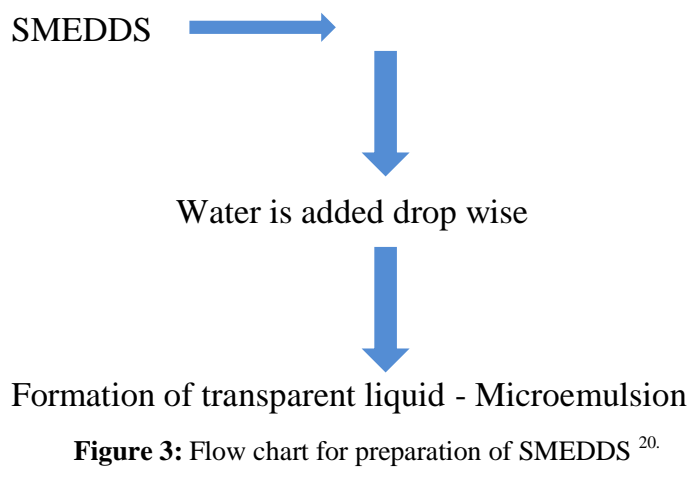

\section{EVALUATION OF SMEDDS}

The efficiency of self-micro-emulsification could be estimated by determining the evaluation parameter.

\section{Droplet size and particle size measurement:}

The particle size of the micro emulsion is determined by photon correlation spectroscopy or SEM (Scanning Electron Microscopy) which can measure sizes between 10 and $5000 \mathrm{~nm}$. The Nano metric size range of the particle is retained even after 100 or 1000 times diluted with distilled 
water, which proves the system's compatible with excess water ${ }^{2}$.

\section{Refractive index and percent transmission:}

Refractive index and percent transmittance proves the clearness of formulation. The refractive index of the SMEDDS is measured by refractometer and compared with that of water. The percent transmittance of the system is measured at particular wavelength using UV-visible spectrophotometer keeping distilled water as blank, if refractive index of system should be similar to that of water. Formulation showing transmittance $>99$ percent is transparent in nature ${ }^{2,4}$.

\section{Zeta potential measurement:}

Zeta potential for micro emulsion can be determined using a suitable Zeta sizer, in triplicate samples ${ }^{19}$.

\section{Stability:}

SMEDDS was diluted with distilled water and to check the temperature stability of samples, they were kept at two different temperature range $\left(2-8^{\circ} \mathrm{C}\right.$ (refrigerator), room temperature) and observed for any evidences of phase separation, flocculation or drug precipitation. In order to estimate metastable systems, the optimized SMEDDS formulation was diluted with distilled water. Then micro emulsion was centrifuged at $1000 \mathrm{rmin}-1$ for $15 \mathrm{~min}$ at $37^{\circ} \mathrm{C}$ and observed for any alteration in homogeneity of micro emulsions ${ }^{24}$.

\section{Centrifugation:}

Passed formulations are centrifuged thaw cycles between $21{ }^{\circ} \mathrm{C}$ and $+25{ }^{\circ} \mathrm{C}$ with storage at each temperature for not less than $48 \mathrm{~h}$ is done at $3500 \mathrm{rpm}$ for $30 \mathrm{~min}$. Those formulations that does not show any phase separation are taken for the freeze thaw stress test.

\section{In vitro release study:}

In vitro drug release study of SMEDDs formulation was performed by dialysis method, dissolution apparatus 2 and diffusion cell. Study of drug release was done by modified diffusion cell in $200 \mathrm{ml}$ buffer solution $6.8 \mathrm{pH}$. $1 \mathrm{gm}$ SMEDDs formulation was placed in boiling tube, both side of boiling tube was opened and one side of tube was tied with cellophane membrane and dipped in buffer solution kept in a beaker below. Upper side of the cylinder was clamped to hold. The beaker was continuously stirred by magnetic stirrer and sample was withdrawn after different time intervals it in straight position and analyzed by UV Spectrophotometer \% drug dissolved at different time intervals was calculated using the beer Lambert's equation.

\section{Bioavailability study:}

Based on the self-emulsification properties, particle size data and stability of micro emulsion the formulation is selected for bioavailability studies. The in vivo study is performed to quantify the drug after administration of the formulation. Pharmacokinetic parameters of the maximum plasma concentration ( $\mathrm{C} \max$ ) and the corresponding time ( $\mathrm{T} \max$ ) for the drug following oral administration are calculated. The relative Bioavailability (BA) of SMEDDS form to the conventional table is calculated using the following Equation Relative BA $(\%)=($ AUC test/AUC reference) $\mathrm{X}$ (Dose reference/Dose test).

\section{Dispersibility test:}

The efficiency of self-emulsification of oral Nano or micro emulsion is assessed using a standard USP XXII dissolution apparatus 2. One milliliter of each formulation is added to $500 \mathrm{ml}$ of water at $37 \pm 0.5^{\circ} \mathrm{C}$. A standard stainless steel dissolution paddle rotating at $50 \mathrm{rpm}$ provides gentle agitation. The in vitro performance of the formulations is visually assessed using the following grading system 16 . Grade A: Rapidly forming (within $1 \mathrm{~min}$ ) Nano emulsion having a clear or bluish appearance. Grade B: Rapidly forming slightly less clear having a bluish-white appearance. Grade C: Fine milky emulsion that forms within 2 min. Grade D: Dull grayish white emulsion having slightly oily appearance that is slow to emulsify. Grade E: Formulation exhibiting either poor or minimal emulsification with large oil globules present on the surface. Grade A and Grade B formulation will remain as Nano emulsion when dispersed in GIT. While formulation falling in Grade C could be recommended for SMEDDS formulation $^{2,11,24}$.

\section{CONCLUSION}

SMEDDS is an incredible and valuable methodology for the preparation of drug compound with poor solubility, presystemic first-pass impact, high atomic weight, gastric irritation, enzymatic degradation, low rate of dissolution, and low bioavailability. This methodology is appropriate for all medications of BCS since arranged emulsion gives quicker absorption, faster dissolution rates, and high bioavailability because of solubilization of medication in lipidic excipients which dodges the dissolution step. This methodology needs more misuse in field of SEOPT plans and other dosage form. They need IVIVC which should be broad and constant correlation these investigations in not so distant utilizing bio relevant media.

\section{REFERENCE}

1. Akula S, Gurram AK, and DevireddySR. Review on:Self-MicroEmulsifying Drug Delivery System: An Attractive Stratergy for Enhance Therapeutic Profile. International Scholarly Research Notices, 2014; 11(8): 1-4.

2. Deshmukh AS. Review on:Recent Advances in Self Emulsifying Drug Delivery System. International Journal of Pharmaceutical Science and Nanotechnology (IJPSN), 2015; 8(1): 3-4.

3. Deshmukh AS, Mahajan VR. Review on: Advanced Delivery of Poorly Water Soluble Drug By Lipid Based Formulation as (SMEDDS). Asian Journal of Research in Biological and Pharmaceutical Science, 2015; 3(1):15-18.

4. Deshmukh AS, Mahajan VR. Review on: Advanced Delivery of Poorly Water Soluble Drug Atrovastain By Lipid Based Formulation as (SMEDDS). Asian Journal of Pharmaceutical Research and Development, 2015; 3(2):22-29.

5. Dokania S, Joshi AK. Review on:Self-Micro-Emulsifying Drug Delivery System (SMEDDS) - Challenges and Road Ahead. Drug Delivery Informa Healthcare, 2014; 22(6):677.

6. Gadhe LT, Kapadi SV, Bachkar B, Gandhi M Review on: Recent Patent Review on Self-Micro-Emulsifying Drug Delivery System. World Journal of Pharmaceutical Research, 2015; 4(3):970. 
7. Gahlawat N, Verma R, Kaushik D. Review on: Recent Development in Self-Micro-Emulsifying Drug Delivery System: An Overview. Asian Journal of Pharmaceutics, 2019; 13(1):59- 60.

8. Gautam P, Shastri D. Review on: Self -Micro Emulsifiying Drug Delivery System to enhance the solubility of the hydrophobic drugs. Current Trends InBiomedical Engineering and Biosciences (JP Juniprer), 2018; 13(4):60.

9. Gupta RN, Gupta R, Rathore GS. Review on: Enhancement of Oral Bioavailability of Lipophilic Drug Delivery System (SMEDDS). International Journal of Drug Development and Research, 2009: 1(1):13.

10. Jaiswal JJ, Solanki AJ, Yadav SK. Review on: A Review on Solid Self Micro Emulsifying Drug Delivery System: Formulation Strategies to Improve the Bioavailability of Poorly Soluble Drug. Journal of Pharmaceutical Science and Bioscientific Research (JPSBR), 2016; 6(3):241.

11. Kalamkar P, Pawar K, Baddi H, Thawkar B, Yevale R, Dr. Kale M “ Self- Micro-Emulsifying Drug Delivery System (SMEDDS). International Journal of Pharmacy and Pharmaceutical Research (IJPPR).2016; 6(3): 370

12. Khedekar K, Mittal S. Review on: Self -Micro-Emulsifying Drug Delivery System. International Journal Pharmaceutical Science and Research.2013; 4 (12):4497- 4498.

13. Maurya SD, Arya RK, Rajpal G, Dhakar RC. Review on: Self-MicroEmulsifying Drug Delivery Systems (SMEDDS): A Review on Physico-Chemical and Biopharmaceutical Aspect. Journal of Drug Delivery and Therapeutics, 2017; 7(3):59- 60

14. Pattewar SV, Kasture S, Pande V, Sharma S. Review on: Self-MicroEmulsifying Drug Delivery System: A Lipid Based Drug Delivery System. International Journal of Pharmaceutical Sciences and Research, 2016; 7(2): 443-448.

15. Pawar SD, Gujrathi NA, Rane BR, Pawar SP. Review on: Self-MicroEmulsifying Drug Delivery System (SMEDDS): A Promising Drug Delivery System for Enhancement of Bioavailability. Indian Journal of Drug, 2016; 4(3):6-7.

16. Potphode VR, Deshmukh AS, Mahajan VR. Review on; Self-MicroEmulsifying Drug Delivery System: Approach for Enhancement of
Bioavaibility of Poorly Water Soluble Drugs. Asian J. Pharma Tech, 2016; 6(3): 63-65.

17. P. Reddy UM, P. Babu R, P. Rao M, Edukoudalu V, Malikharjunarao KR. A Review on: Self-Micro Emulsifying Drug Delivery System. International Journal of Research in Pharmaceutical and Nano Sciences, 2013; 2(3):319.

18. P. Tripura S, P. Mounika. Formulation and Evaluation of SMEDDS Containing Febuxostat By Employing Arachis Oil and Labrasol As Oil and Surfactant System. World Journal of Pharmaceutical Research, 2018; 7(11): 543- 545.

19. 19. Reddy S, Katyayani T, Navatha, A, Ramya G. Review on SelfMicro-Emulsifying Drug Delivery System. International Journal of Research in Pharmaceutical Sciences, (2011); 2(3):383- 386.

20. Sharma VK., Koka A, Yadar J, Sharma AK., Keservani RK. Review on: Self-Micro-Emulsifying Drug Delivery System: A Strategy to Improve Oral Bioavailability. Ars Pharmaceutical, 2016; 57(3):102, 99.

21. Sharma V, Singh J, Gill B, Harikumar SL. Review on: SMEDDS: A Novel Approach For Lipophilic Drugs. International Journal Pharmaceutical Sciences and Research, 2012; 3(8):2441-2444.

22. Thakare P, Mogal V, Borse P, Dusane J, Kshirsagar S. Review on: Self-Micro-Emulsified Drug Delivery System. Pharmaceutical and Biological Evaluations,2016; 3 (2):146- 147.

23. V.Kaushik, S. Preethi, P. Satish, Uma Shankar MS, Thakur RS. Development of Solid Micron Emulsifying Drug Delivery System. International Journal of Pharmaceutical Science and Nanotechnology (IJPSN),2013; 6 (2):2014-2015.

24. Wankhade VP, Kale NS, Tapar KK. Review on: Self Micro Emulsifying Nutraceutical and Drug Delivery Systems. International Journal of Pharmaceutical Science and Nanotechnology (IJPSN), 2014; 7(3): 2521-2526.

25. Wu L, QiaoY, Wang L, Guo J, Wang G, He W, Yin I, ZhaoJ. Review on: A Self-Micro-Emulsifying Drug Delivery System (SMEDDS) for a Novel Meditative Compound against Depression: A preparation and Bioavailability study in rats. American association of pharmaceutical scientists (AAPS), 2014; 16(5):1052-1054. 